\title{
Identificação Precoce do Risco para Transtornos da Atenção e da Leitura em Sala de Aula
}

\author{
Olga Valéria Andrade ${ }^{1}$ \\ Paulo Estêvão Andrade \\ Simone Aparecida Capellini \\ Colégio Criativo \\ Universidade Estadual Paulista
}

\begin{abstract}
RESUMO - Testou-se a acurácia de atividades pedagógicas coletivas, baseadas em julgamentos fonológicos por meio do pareamento entre figuras e de figuras com palavras faladas, na identificação de escolares de risco para transtornos da atenção e da leitura em sala de aula. Quarenta e cinco escolares do $2^{\circ}$ ano (idade média de 7 anos, 29 do gênero masculino), foram divididos em grupo controle, sem dificuldade de leitura-escrita $(n=32)$, e grupo de risco, com dificuldade de leitura $(n=13)$. O baixo desempenho nessas atividades, definido como os escores acima de 1,65 DP abaixo da média do grupo controle, apresentou boa sensitividade (verdadeiros positivos) e especificidade (verdadeiros negativos) na identificação precoce dos escolares de risco.
\end{abstract}

Palavras-chave: dislexia, transtornos de aprendizagem, educação

\section{Early identification of attention and reading disorders in the classroom}

\begin{abstract}
In this his study the accuracy was investigated of collective pedagogical activities, based on phonological judgments by matching figures and figures to spoken words, to identify students at risk for reading and/or attention disorders. Forty-five second graders (mean age of 7 years, 29 males) were divided into two groups, a control group, without reading difficulties $(n=32)$, and an at-risk group, with reading difficulties $(n=13)$. The low-achievement on these collective activities, defined by scores more than $1.65 \mathrm{SD}$ below the mean of the control group, presented good sensitivity (true positives) and specificity (true negatives) in the early identification of at risk students.
\end{abstract}

Keywords: dyslexia, learning disorders, education

Os problemas de aprendizagem ou baixo rendimento escolar (BRE) podem ser definidos como um rendimento aquém do esperado nos domínios acadêmicos da leitura-escrita e matemática (Hinshaw, 1992). De acordo com Ciasca (2004), 30\% a 40\% dos escolares nos primeiros anos do ensino fundamental podem apresentar BRE.

Por meio de questionários objetivos e específicos para pais e professores, estudos mais recentes no Brasil sinalizam que $30 \%$ dos escolares do $1^{\circ}$ ao $5^{\circ}$ ano do ensino fundamental, tanto na rede pública quanto particular, apresentam queixas de BRE em relação ao seu grupo-classe (Alves \& Ribeiro, 2011; Bicalho \& Alves, 2010). Esse número é um pouco menor do que o reportado nos Estados Unidos da América, onde aproximadamente $36 \%$ dos estudantes no $4^{\circ}$ ano lêem abaixo dos níveis básicos (Shaywitz, Morris, \& Shaywitz 2008).

Entretanto, é importante diferenciar entre BRE resultante de fatores ambientais, extrínsecos ao estudante, tais como fatores pedagógicos, socioeconômicos, culturais e emocionais, e BRE decorrente de fatores de origem genético-neurológica, principalmente disfunções congênitas do sistema nervoso central, intrínsecos ao escolar. O primeiro é, de forma geral, denominado de dificuldades de aprendizagem, ao passo que

1 Endereço para correspondência: Colégio Criativo - Unidade Tangará: Rua das Safiras, 45. Marília, SP. CEP: 17516-130.

E-mail:sacap@uol.com.br o segundo BRE tende a ser permanente e é nomeado como transtorno ou distúrbio da aprendizagem (Alves, Mousinho, \& Capellini, 2011; Ciasca, 2004).

Segundo a definição da quarta versão do Manual de Diagnóstico e Estatística de Transtornos Mentais (DSM-IV, APA, 2002), transtorno da aprendizagem (TA) é um termo amplo que inclui vários distúrbios distintos, alguns específicos, caracterizados por um baixo rendimento inesperado "em testes padronizados e individualmente administrados de leitura, matemática ou expressão escrita" (p. 44). O TA é inesperado porque ocorre apesar de inteligência normal, adequadas oportunidades educacionais, socioeconômicas e afetivas, bem como ausência de transtornos sensoriais e neurológicos aparentes.

Os TAs incluem: a) transtorno da leitura ou dislexia (sendo ambos os termos intercambiáveis), caracterizado por prejuízos mais restritos à linguagem escrita; b) transtorno da matemática, também referido como transtorno específico de habilidades aritméticas e discalculia do desenvolvimento (DD); c) transtorno da expressão escrita (comprometimento da ortografia e caligrafia, mas geralmente associado a outros transtornos); e d) transtorno da aprendizagem sem outra especificação, normalmente referido no Brasil distúrbio de aprendizagem (Ciasca, 2004), caracterizado por prejuízos mais gerais envolvendo a linguagem oral, a linguagem escrita e a matemática (APA, 2002). Em comparação às dificuldades de aprendizagem, a prevalência de TA é bem menor, porém 
similar entre os países, em torno de 2 a $10 \%$ da população estudantil (APA, 2002).

A maioria dos pesquisadores concorda que os transtornos de aprendizagem são muito mais fáceis de prevenir do que remediar e que a intervenção linguística e de leitura-escrita precoce previne ou reduz significativamente os potenciais problemas de leitura. Portanto, atualmente enfatiza-se que a identificação de pré-escolares e escolares leitores iniciantes com prejuízos nas habilidades relacionadas à leitura-escrita é um procedimento crucial (Alves, Siqueira, Lodi, \& Araújo, 2011; Catts, Fey, Zhang, \& Tomblin, 2001; Fuchs \& Fuchs, 2006; Shaywitz et al., 2008).

Porém, a identificação e intervenção precoces são difíceis de operacionalizar com base nos modelos diagnósticos baseados na discrepância QI-rendimento, atualmente predominantes no diagnóstico de transtorno de aprendizagem, e os quais têm sido duramente criticados (Dombrowski, Kamphaus, \& Reynolds, 2004; Fletcher \& Vaughn, 2009; Vellutino, Fletcher, Snowling, \& Scanlon, 2004). Os modelos de discrepância QI-rendimento tiveram origem na noção de inesperado, central ao conceito de transtornos de aprendizagem (Fletcher \& Vaughn, 2009; Shaywitz \& Shaywitz, 2008). Tais modelos requerem que o rendimento em um dado domínio acadêmico (com base em testes referenciados em normas) esteja a um ou dois desvios-padrão abaixo da média do QI geral para que o escolar seja diagnosticado e encaminhado para programas de intervenção (Dombrowski et al., 2004; Fuchs \& Fuchs, 2006; Shaywitz et al., 2008). Os modelos de discrepância QI-rendimento têm sido criticados não relativamente à noção de inesperado, "mas sim com o efeito prático na vida real de se implementar este modelo em um ambiente de escola primária" (Shaywitz et al., 2008, p. 454), pois deve-se aguardar até o $4^{\circ}$ ano para que as eventuais discrepâncias entre desempenho acadêmico e QI apareçam e o diagnóstico seja feito.

A espera pelo surgimento de uma eventual discrepância QI-rendimento no $4^{\circ}$ ano, levou alguns autores a cunharem esses modelos de wait-to-fail (espera pelo fracasso) (Fuchs $\&$ Fuchs, 2006), porque priva o escolar com BRE de receber as intervenções remediativas adequadas a suas dificuldades em períodos de maior plasticidade neurocognitiva e, portanto, mais propícios para a intervenção, isto é, no início da alfabetização ou até mesmo antes. Essa espera pelo fracasso também gera uma lacuna escolar (defasagem em relação ao grupo-classe) maior e mais difícil de ser superada (Andrade, Prado, \& Capellini, 2011; Fuchs \& Fuchs, 2006).

Nesse contexto é fundamental que se desenvolvam ferramentas de triagem que sejam rápidas e de fácil aplicação pelos professores, eficientes e de baixo custo e, ao mesmo tempo, tenham boa sensitividade (percentual de verdadeiros positivos) e boa especificidade (percentual de verdadeiros negativos), critérios comumente citados na literatura para medir a precisão das ferramentas de identificação precoce (Catts et al., 2001; Vellutino, Scanlon, Zhang, \& Schatschneider, 2008). Porém, esse tipo de produção ainda é muito escassa na literatura nacional (Andrade et al., 2011; Navas, 2011). Não menos importante é o papel de um pré-diagnóstico realizado pelos professores de forma competente e assertiva (Capellini \& Conrado, 2009; Capellini, Tonelotto, \& Ciasca, 2004) por meio de avaliações baseadas no currículo (Dombrowski et al., 2004; Fuchs \& Fuchs, 2006), tal como temos no Brasil os procedimentos baseados no conceito de avaliação diagnóstica (Luckesi, 2005).

Do ponto de vista prático da identificação de escolares de risco para dislexia, devemos destacar que a própria classificação proposta pelo DSM-IV (APA, 2002) indica que os prejuízos de leitura correspondem a um sintoma comum compartilhado pela maioria dos transtornos. De fato, vários autores apontam que mais de $80 \%$ dos diferentes tipos de TA compartilham prejuízos de leitura (Fuchs \& Fuchs, 2006; Shaywitz et al., 2008).

Embora as definições mais clássicas considerem o TL (ou dislexia) um transtorno específico da aquisição e do desenvolvimento da leitura (principalmente fluência e acurácia) decorrente de um déficit nas habilidades de processamento fonológico (consciência fonológica, nomeação rápida e memória de trabalho fonológica) (Alves et al., 2011; Shaywitz \& Shaywitz, 2008; Vellutino et al., 2004), evidências recentes sugerem que déficits de atenção também são um importante fator causal do TL (Shaywitz \& Shaywiz, 2008). A inatenção geralmente está associada a prejuízos de nomeação rápida (RAN) e memória de trabalho verbal (MTF), independentemente de prejuízos na consciência fonológica (CFO) (Shanahan et al., 2006; Willcutt et al., 2010). Severos déficits de nomeação, sem qualquer outro sintoma associado, também podem ser uma causa independente da dislexia, conforme a hipótese do duplo-déficit (Wolf \& Bowers, 1999).

Embora o transtorno do déficit de atenção/hiperatividade (TDA/H), transtorno neuropsiquiátrico mais comum na infância, não seja considerado um TA, os subtipos desatento (predomínio da desatenção ou TDA) e combinado (hiperatividade com inatenção ou TDAH) geralmente estão associados a dificuldades na aquisição e desenvolvimento da linguagem escrita (Siqueira et al., 2011). Hoje se reconhece que a desatenção (ou inatenção), evidenciada principalmente por prejuízos na memória de trabalho visuoespacial (MTVE) (Castellanos, Sonuga-Barke, Milham, \& Tannock, 2006; Martinussen \& Tannock, 2006), está frequentemente associada a prejuízos de RAN e MTF, que são sintomas também presentes na dislexia (Martinussen \& Tannock, 2006; Willcutt et al., 2010). Em suma, embora sejam mais proeminentes na dislexia do que no TDAH, os prejuízos em RAN e MTF são sintomas comuns subjacentes à leitura deficiente no TDA/H, independentemente de comorbidade entre os dois transtornos (Martinussen \& Tannock, 2006; Willcutt et al., 2010).

Com base na breve revisão apresentada, sugerimos que o risco para TL deveria ser abordado em termos de suas principais causas subjacentes, isto é, prejuízos nas habilidades de CFO, RAN e MTF, independentemente do tipo de transtorno do qual esses prejuízos fazem parte, se dislexia, distúrbio de aprendizagem ou TDAH. Portanto, o presente estudo investigou a sensitividade (percentual de verdadeiros positivos ou a acurácia na identificação de escolares de risco) e especificidade (percentual de verdadeiros negativos ou a acurácia na identificação de escolares fora de risco) de algumas atividades pedagógicas coletivas na identificação precoce de escolares de risco para transtornos de atenção e de aprendizagem. Essas atividades incluem julgamentos 
fonológicos a partir do pareamento entre figuras e de figuras com palavras denominadas de ferramentas alternativas do educador (FAE), em consonância com Andrade et al. (2011), bem como a prova de escrita de palavras e pseudo palavras do Teste de Desempenho Cognitivo-Linguístico (Capellini \& Smythe, 2008).

\section{Método}

\section{Participantes}

Nesta pesquisa de natureza longitudinal, com delineamento de caso controle, participaram 45 escolares com idade média de 7 anos e 4 meses, sendo 29 do gênero masculino e 16 do gênero feminino, provenientes de quatro salas de aula de uma escola da rede particular de ensino da cidade de Marília, São Paulo, e que na fase inicial do estudo estavam no início do $2^{\circ}$ ano do ensino fundamental. Todos os participantes eram provenientes da classe média-alta, cujo status socioeconômico correspondia às classes $\mathrm{A}$ e $\mathrm{B}$, de acordo com a Escala para Avaliação do Status Socioeconômico da Associação Brasileira dos Institutos de Pesquisa de Mercado - ABIPEME (Almeida \& Wickerhauser, 1991). A escolha por alunos da classe média-alta objetivou minimizar o peso de aspectos socioeconômicos, culturais (Demo, 2007) e pedagógicos (Capellini et al., 2009) sobre o desempenho escolar, fatores associados à maior ocorrência de problemas acadêmicos na rede pública em comparação à particular (Alves \& Ribeiro, 2011; Bicalho \& Alves, 2010; Demo, 2007; Silva \& Santos, 2011).

Foram entregues 69 termos de consentimento correspondentes a todos os alunos de quatro salas de aula do $2^{\circ}$ ano do ensino fundamental, dos quais 24,6\% (17 escolares) apresentavam queixas de dificuldades de aprendizagem de acordo com o prontuário escolar, que incluiu informações dos pais ou responsáveis e a avaliação diagnóstica realizada pelos professores (Luckesi, 2005). Dos 69 termos de consentimento entregues, 45 retornaram assinados, dos quais $28,8 \%$ ( 13 escolares) faziam parte do grupo com queixas de dificuldade de aprendizagem. Os critérios para inclusão foram: a) a assinatura pelos pais ou responsáveis do Termo de Consentimento Livre e Esclarecido; b) os escolares apresentarem acuidade visual, auditiva e desempenho cognitivo dentro dos padrões de normalidade e nenhum histórico de danos neurológicos descrito no prontuário escolar. Os critérios de exclusão foram: a) a ausência da assinatura do Termo Livre e Esclarecido; b) suspeita de déficits sensoriais (auditivo e/ou visual) não corrigidos; c) histórico de problemas neurológicos adquiridos; d) portadores de necessidades educativas especiais, em regime de inclusão.

\section{Material}

Avaliação Diagnóstica (AD). Todos os escolares do presente estudo participaram da avaliação diagnóstica. Para o propósito específico da presente investigação focamos unicamente nas avaliações das habilidades de leitura e escrita
(Fletcher \& Vaughn, 2009; Fuchs \& Fuchs, 2006) descritas a seguir:

1) Leitura: fluência e acurácia na leitura em voz alta de palavras simples monossílabas, dissílabas e trissílabas extraídas dos livros didáticos e apostilas usados pela escola.

2) Leitura silenciosa: desenhar o significado de uma palavra escrita.

3) Escrita: escrever todas as letras do alfabeto que conhece (letra de forma ou cursiva), fazer um círculo vermelho em volta das vogais e um círculo azul das consoantes, escrever a letra com a qual começa o nome de uma figura apresentada.

4) Conhecimento de sílabas: escrever a sílaba correta para completar uma palavra.

5) Conhecimento de palavras: formar, usando o alfabeto móvel, o maior número possível de palavras que conhece e depois escrevê-las no papel.

6) Escrita: escrever palavras ditadas pelo examinador.

7) Compreensão da linguagem oral: ao acabar de ouvir uma história, desenhar o que compreendeu.

Teste de Desempenho Cognitivo-Linguístico (TDCL) (Capellini \& Smythe, 2008).

1. Versão coletiva. Três subtestes foram aplicados em todos os participantes: a) Conhecimento do alfabeto (ALF): letras do alfabeto que o escolar conhece e pode escrever (escore máximo: 26); b) Cópia de formas (COP): copiar um círculo, quadrado e losango e uma forma complexa combinando elementos destas figuras simples (escore máximo de acordo com escala padronizada: 7); c) Escrita sob ditado (ESC): 30 palavras reais e 10 pseudopalavras ditadas pelo examinador (escore máximo: 40).

2. Versão individual. Consiste em cinco testes que avaliam: a) Leitura (LEIT): leitura de palavras e pseudopalavras: fluência (palavras corretamente lidas em um minuto) e acurácia (palavras corretamente lidas de uma lista de 70 palavras; b) Consciência fonológica (CFO): identificação de rima (de três palavras ouvidas dizer quais as duas que rimam), aliteração (de três palavras ouvidas dizer quais as que começam com o mesmo som) e segmentação silábica (bater uma palma para cada sílaba de uma palavra ouvida); c) Processamento auditivo: memória de trabalho fonológica (MTF) (repetição de sete sequências contendo de duas a cinco palavras); d) Processamento visual: inclui a memória de trabalho visual para formas (MV) (reproduzir, na mesma ordem mostrada pelo examinador, sequências de duas a cinco figuras com detalhes) e memória de trabalho visuoespacial (MVE) (número de erros de rotação cometidos na tarefa de memória visual); e) Velocidade de processamento: nomeação rápida de figuras e dígitos (RAN). Para maiores detalhes, veja Capellini e Smythe (2008). Nas Tabelas 2 e 3 essas provas são referidas somente pelas respectivas siglas.

A versão coletiva do TDCL foi aplicada durante as aulas, com duração de aproximadamente 50 minutos. Os alunos receberam explicações e treino, garantindo o entendimento em cada subteste, bem como organização e comportamento durante os testes (não levantar do lugar nem fazer perguntas 
durante o teste, por exemplo). Na versão individual do TDCL, cada aluno foi recebido em uma sala bem iluminada com o mínimo de ruído possível, considerando o ambiente escolar, e cada sessão teve duração de aproximadamente 40 a 60 minutos. Todas as provas foram precedidas de explicação e treino, conforme orientado no TDCL (Capellini \& Smythe, 2008).

Ferramentas Alternativas do Educador (FAE) (Andrade et al., 2011). Constituem um conjunto de seis tarefas coletivas, baseadas nas tarefas de processamento fonológico do Teste de Desempenho Cognitivo-Linguístico (Capellini \& Smythe, 2008), elaboradas de modo a envolverem a nomeação, memória de trabalho fonológica e consciência fonológica e serem facilmente aplicáveis coletivamente em sala de aula pelo professor.

No presente estudo utilizamos somente as quatro FAE que apresentaram as mais fortes e significantes correlações em estudo prévio (Andrade et al., 2011), a saber: a) FAE 1 de identificação de aliteração das palavras que compartilham o fonema inicial: das três figuras escolha as duas que tem o mesmo som no começo, marcando um X em cada figura (escore máximo 10); b) FAE 2 de identificação de rima (compartilhamento do som final no nível intra-silábico): das três figuras marque um $\mathrm{X}$ nas duas figuras que rimam ou terminam com o mesmo som (escore máximo 10); c) FAE 5 de identificação de rima (compartilhamento do som final no nível intrassilábico): das três figuras, marque um X embaixo daquela que rima com a palavra falada (escore máximo 10); e d) FAE 6 de identificação de aliteração das palavras que compartilham o fonema inicial: coloque um X embaixo da figura que começa com o mesmo som da palavra falada (escore máximo 10).

\section{Procedimento}

Após o recebimento dos termos de consentimento livre e esclarecido, foram feitas a análise do prontuário escolar dos participantes (Luckesi, 2005) do último bimestre do ano anterior (quando os participantes cursavam o $1^{\circ}$ ano) e a Avaliação Diagnóstica (AD) realizada no primeiro bimestre do ano letivo em que se iniciou o estudo, quando os alunos cursavam o $2^{\circ}$ ano, a qual será referida como $\mathrm{AD}^{\mathrm{I}}$. No início do segundo bimestre do $2^{\circ}$ ano, foram realizadas a primeira medição do Teste de Desempenho Cognitivo-Linguístico $\left(\mathrm{TDCL}^{\mathrm{I}}\right.$ ) nas versões coletiva e individual (Capellini \& Smythe, 2008) e as atividades FAE. Um ano após o início do estudo, no primeiro bimestre do $3^{\circ}$ ano do ensino fundamental, novamente realizou-se a avaliação diagnóstica $\left(\mathrm{AD}^{\mathrm{II}}\right)$ e aplicou-se o Teste de Desempenho Cognitivo-Linguístico (TDCL ${ }^{\mathrm{II}}$ ). Porém, por indisponibilidade de horário da escola e dos professores, só foi possível aplicar as provas de leitura (LEIT $\left.{ }^{\mathrm{II}}\right)$, consciência fonológica $\left(\mathrm{CFO}^{\mathrm{II}}\right)$, memória de trabalho fonológica $\left(\mathrm{MTF}^{\mathrm{II}}\right)$ e nomeação rápida (RAN ${ }^{\mathrm{II}}$ ) do TDCL.

Com base nos procedimentos descritos, os escolares com BRE foram selecionados em dois grupos de risco de referência, um constituído pelos escolares em defasagem acadêmica de avaliações diagnósticas $\mathrm{AD}^{\mathrm{I}}$ e $\mathrm{AD}^{\mathrm{II}}$ e referidos como $\mathrm{GD}^{\mathrm{I}}$ e $\mathrm{GD}^{\mathrm{II}}$, e o outro constituído pelos grupos de risco específicos de tarefa ou experimentais (GREs) formados pelos escolares com baixo rendimento nas provas do TDCL ${ }^{\mathrm{I}}$, TDCL ${ }^{\mathrm{II}}$ e nas atividades FAE (somente uma medição no início do $2^{\circ}$ ano). Conforme indica a Tabela 2 , foram selecionados dois grupos de risco de referência.

Tabela 1. Características dos Desempenhos do Grupo Total e Grupos Controles Específicos de Tarefa (GCE) no TDCL ${ }^{\mathrm{I}}$ TDCL ${ }^{\mathrm{II}}$ e FAE

\begin{tabular}{|c|c|c|c|c|c|c|c|c|c|}
\hline \multirow[b]{2}{*}{ Testes } & \multicolumn{4}{|c|}{ Grupo Total } & \multicolumn{4}{|c|}{$\mathrm{GCE}$} & \multirow[t]{2}{*}{ Corte } \\
\hline & $n$ & $M$ & $D P$ & $\mathrm{LIC}$ & $n$ & $M$ & $D P$ & $\mathrm{LIC}$ & \\
\hline Alfabeto (ALF) & 45 & 24,47 & 2,72 & 23,65 & 36 & 25,56 & 0,65 & 25,33 & $\leq 24$ \\
\hline Leitura (LEIT) & 45 & 105,09 & 28,68 & 96,47 & 31 & 120 & 12,03 & 115,58 & $<100$ \\
\hline LEIT $^{\text {II }}$ & 45 & 125,90 & 24,05 & 118,31 & 33 & 136,9 & 18,68 & 129,79 & $<109$ \\
\hline Escrita (ESC) & 45 & 30,09 & 8,33 & 27,58 & 33 & 34,15 & 3,07 & 33,06 & $<29$ \\
\hline Leitura/Escrita (LE) & 45 & 135,18 & 36,18 & 124,31 & 31 & 151,41 & 15,93 & 145,85 & $<131$ \\
\hline Consc. Fonológica (CFO) & 45 & 36,24 & 4,48 & 34,90 & 31 & 38,65 & 2,28 & 37,81 & $<35$ \\
\hline $\mathrm{CFO}^{\mathrm{II}}$ & 41 & 37,29 & 5,15 & 35,66 & 32 & 38,84 & 2,01 & 38,12 & $<36$ \\
\hline Mem. Trab. Fonol. (MTF) & 45 & 3,76 & 1,15 & 3,41 & 40 & 4 & 0,96 & 3,69 & $<3$ \\
\hline $\mathrm{MTF}^{\mathrm{II}}$ & 41 & 4,37 & 1,13 & 4,01 & 33 & 4,76 & 0,86 & 4,45 & $\leq 3$ \\
\hline Cópia de Formas (COP) & 45 & 4,84 & 2,13 & 4,2 & 33 & 5,91 & 1,28 & 5,45 & $<4$ \\
\hline Mem.Visual (MV) & 45 & 5,62 & 1,13 & 5,28 & 38 & 5,95 & 0,89 & 5,65 & $<5$ \\
\hline Mem. Visuoespacial (MVE) & 45 & 2,40 & 3,12 & $3,34^{\mathrm{a}}$ & 35 & 0,97 & 0,89 & $1,28^{\mathrm{a}}$ & $\geq 3$ \\
\hline Nomeação Rápida (RAN) & 45 & 83,98 & 18,16 & $78,52^{\mathrm{a}}$ & 33 & 75,79 & 8,79 & $78,91^{\mathrm{a}}$ & $>90$ \\
\hline $\mathrm{RAN}^{\mathrm{II}}$ & 41 & 73,22 & 13,61 & $68,92^{\mathrm{a}}$ & 29 & 66,69 & 8,81 & $70,04^{\mathrm{a}}$ & $>81$ \\
\hline FAE & 45 & 32,16 & 4,93 & 30,74 & 27 & 35,07 & 2,30 & 34,21 & $<32$ \\
\hline
\end{tabular}

Nota. TDCL (Teste de Desempenho Cognitivo-Linguístico); FAE (Ferramentas Alternativas do Educador, aplicadas somente no início do estudo); ${ }^{\text {I }}$ (primeira aplicação no início do estudo); "I (segunda aplicação, um ano depois); LIC (limite inferior do intervalo de confiança de 95\%); Corte ( ponto de corte de 1,65

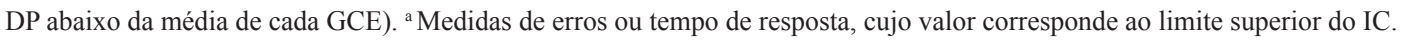


Um foi constituído pelos escolares com BRE de fato, isto é, em defasagem acadêmica conforme as avaliações diagnósticas $\mathrm{AD}^{\mathrm{I}}$ e $\mathrm{AD}^{\mathrm{II}}$ e referidos como $\mathrm{GD}^{\mathrm{I}}$ e $\mathrm{GD}^{\mathrm{II}}$. O outro foi constituído pelos grupos de risco específicos de tarefa ou experimentais (GREs) formados pelos escolares com baixo rendimento nas provas do TDCL ${ }^{\mathrm{I}}$, TDCL ${ }^{\mathrm{II}}$ e nas atividades FAE (somente primeira medição)

Entretanto, no que se refere aos grupos de risco, obtidos com a TDCL ${ }^{\text {II }}$ (ver Tabela 3), exceto a prova de escrita, não foram discutidos em termos de seu potencial como ferramentas de identificação, mas apenas como dados adicionais e importantes para ratificar o status de risco dos escolares identificados pela $\mathrm{AD}^{\mathrm{I}}$ e TDCL ${ }^{\mathrm{I}}$, e também para discussão e interpretação dos dados. A seguir, os critérios e procedimentos para obtenção dos grupos de risco.

Obtenção dos grupos de risco experimentais (GRE). As dificuldades de leitura são normalmente definidas como os escores mais de $1 D P$ abaixo da média, mas outros critérios mais liberais como o percentil 25 (25\% dos escores mais baixos, aproximadamente $0,68 D P$ ) ou 30 (aproximadamente 0,5 DP) ou mais conservadores, como 1,5 DP abaixo da média, são também usados (Catts et al., 2001; Fletcher \& Vaughn, 2009; Fuchs \& Fuchs, 2006). Quanto mais liberal ou mais conservador o critério, maior ou menor número de escolares de risco serão detectados (Catts et al., 2001; Fletcher \& Vaughn, 2009; Fuchs \& Fuchs, 2006). No presente estudo, usou-se o rigoroso critério de desvio sugerido por Ramus et al. (2003) e também utilizado em outros estudos no qual é considerado como baixo rendimento os escores com mais de 1,65 DP abaixo da média do grupo controle para uma dada tarefa.

Primeiramente, foram obtidos grupos controles para cada uma das medidas utilizadas do TDCL e para as FAE separando os participantes com escores acima do limite inferior do intervalo de confiança (LIC) de 95\%, os grupos controles específicos de tarefas (GCE) (ver Tabela 1). Assim, a partir de cada GCE, obtivemos os grupos de risco experimentais (GRE), selecionando-se os escores com ou mais de 1,65 DP abaixo da média obtida de cada GCE (ver Tabela 1), os quais representam os desempenhos iguais ou abaixo do quinto percentil $\left(P_{5}\right)$ (Ramus et al., 2003). Desse modo, foram obtidos GRE não somente para as habilidades de leitura e escrita, mas também para cada prova do TDCL e para as FAE.

O procedimento de se obter grupos de risco específicos de tarefa permitiu não somente testar quais tarefas são mais sensíveis e específicas ao TL, como também investigar quais são os prejuízos cognitivos específicos mais comuns nos escolares de risco para TL. Além disso, emprego de grupos controle específicos de tarefa permitiu verificar as diferenças individuais e o perfil de forças e fraquezas dos alunos de risco, dados essenciais do ponto de vista da intervenção (Reynolds \& Shaywitz, 2009).

Finalmente, a sensitividade das medições do TDCL e das FAE foi calculada considerando-se como verdadeiros positivos o número de escolares dos GRE coincidentes com os do GD (mau desempenho na AD), ao passo que a especificidade foi calculada considerando-se como verdadeiros negativos o número coincidente de escolares fora de risco tanto no GRE quanto no GD.
Abordagem de resposta à intervenção (RI). Os escolares com baixo desempenho na $\mathrm{AD}$, após serem devidamente discutidos e analisados pela equipe pedagógica, foram indicados para as aulas de apoio, um procedimento de rotina da escola e similar aos modelos RI (Fletcher \& Vaughn, 2009; Fuchs \& Fuchs, 2006). Nessa escola, a AD corresponde à avaliação baseada no currículo do modelo RI, a qual identificou um grupo de 13 escolares (28,8 \% da amostra) com significativa defasagem acadêmica, denominado de GD. Quando encaminhados para a intervenção, cada escolar teve suas dificuldades elencadas em uma ficha-prontuário elaborada pelo professor com o objetivo de comunicar e conduzir o planejamento das aulas com esse(s) aluno(s). O planejamento de cada aula foi personalizado, contemplando suas dificuldades e explorando-se estratégias diferenciadas, tanto na sala de aula quanto nas intervenções que se realizavam no turno contrário ao que a criança estudava, com aulas de 60 minutos, duas vezes por semana.

Com a realização da segunda aplicação da $\mathrm{AD}\left(\mathrm{AD}^{\mathrm{II}}\right)$ e do TDCL (TDCL ${ }^{\mathrm{II}}$ ), um ano após o início do estudo, foi possível observar o desenvolvimento do grupo como um todo e o efeito das intervenções pedagógicas no grupo de risco. Isso permitiu verificar quais escolares mostraram responsividade adequada, saindo do grupo de risco, ou inadequada, continuando no grupo de risco para TL.

\section{Análise de Dados}

Toda análise dos dados foi realizada com o programa SPSS (Statistical Package for Social Sciences) em sua versão 16.0. Na análise descritiva foram obtidos a média, o desvio-padrão e o limite inferior do intervalo de confiança do grupo total e dos grupos controles específicos de tarefa (GCE). Os grupos controle específicos de tarefa (GCE) foram obtidos pela separação dos escores acima do limite inferior do intervalo de confiança (LIC) de $95 \%$ em cada tarefa do TDCL e cada uma das atividades FAE. Os grupos de risco (GRE) foram obtidos selecionando-se os escores iguais a, ou mais de 1,65 DP abaixo da média obtida de cada GCE (ver Tabela 1). A sensitividade é quantificada como a razão entre os verdadeiros positivos e a soma dos verdadeiros positivos e falsos negativos (VP/VP $+\mathrm{FN}$ ), ao passo que a especificidade é quantificada como a razão entre verdadeiros negativos e a soma de verdadeiros negativos e falsos positivos (VN/VN + FP) (Vellutino et al., 2008). Um mínimo padrão comumente aceito para a sensitividade é $90 \%$, enquanto um mínimo tolerável para a especificidade é uma porcentagem entre 85 e $90 \%$ (Vellutino et al., 2008).

\section{Resultados}

A primeira avaliação diagnóstica, $\mathrm{AD}^{\mathrm{I}}$, identificou 13 alunos com claras defasagens acadêmicas, referidos como grupo GD. A Tabela 2 mostra os 13 escolares do grupo $\mathrm{GD}^{\mathrm{I}}$, seus escores em cada medição do TDCL ${ }^{\mathrm{I}}$ e das FAE, bem como quais deles possuem escores abaixo do ponto de corte (mais de 1,65 DP abaixo da média do GRE), indicados pelo sobrescrito $\left({ }^{\mathrm{a}}\right)$. 
Tabela 2. Escores dos Escolares em Defasagem Acadêmica $\left(\mathrm{GD}^{1}\right)$ no Teste de Desempenho Cognitivo-Linguístico I (TDCL') e nas Ferramentas Alternativas do Educador (FAE)

\begin{tabular}{|c|c|c|c|c|c|c|c|c|c|c|c|}
\hline \multirow{2}{*}{$\begin{array}{l}\text { Escolares do } \\
\qquad \text { GD }^{\mathrm{I}}\end{array}$} & \multicolumn{10}{|c|}{ Escores no $\mathrm{TDCL}^{\mathrm{I}}$} & \multirow[b]{2}{*}{ FAE } \\
\hline & ALF & LEIT & ESC & LE & $\mathrm{CFO}$ & MTF & COP & MV & MVE & RAN & \\
\hline 04 & $22^{\mathrm{a}}$ & $95^{\mathrm{a}}$ & $16^{\mathrm{a}}$ & $111^{\mathrm{a}}$ & $32^{\mathrm{a}}$ & 4 & $1^{\mathrm{a}}$ & 7 & 1 & 80 & $31^{\mathrm{a}}$ \\
\hline 05 & 26 & $80^{\mathrm{a}}$ & $27^{\mathrm{a}}$ & $107^{\mathrm{a}}$ & $30^{\mathrm{a}}$ & 3 & 7 & 6 & 1 & $100^{\mathrm{a}}$ & $27^{\mathrm{a}}$ \\
\hline 06 & $19^{\mathrm{a}}$ & $37^{\mathrm{a}}$ & $11^{\mathrm{a}}$ & $48^{\mathrm{a}}$ & 36 & $2^{\mathrm{a}}$ & 7 & 5 & $7^{\mathrm{a}}$ & $132^{\mathrm{a}}$ & 35 \\
\hline 10 & 26 & $53^{\mathrm{a}}$ & $20^{\mathrm{a}}$ & $73^{\mathrm{a}}$ & $25^{\mathrm{a}}$ & 3 & 7 & $4^{\mathrm{a}}$ & $4^{\mathrm{a}}$ & 88 & $22^{\mathrm{a}}$ \\
\hline 18 & $21^{\mathrm{a}}$ & $95^{\mathrm{a}}$ & $21^{\mathrm{a}}$ & $116^{\mathrm{a}}$ & $33^{\mathrm{a}}$ & 3 & $4^{\mathrm{a}}$ & 5 & $9^{\mathrm{a}}$ & 88 & 34 \\
\hline 19 & $21^{\mathrm{a}}$ & $90^{\mathrm{a}}$ & $27^{\mathrm{a}}$ & $117^{\mathrm{a}}$ & $25^{\mathrm{a}}$ & $1^{\mathrm{a}}$ & $2^{\mathrm{a}}$ & $4^{\mathrm{a}}$ & $7^{\mathrm{a}}$ & $94^{\mathrm{a}}$ & $26^{\mathrm{a}}$ \\
\hline 22 & $24^{\mathrm{a}}$ & $71^{\mathrm{a}}$ & $28^{\mathrm{a}}$ & $99^{\mathrm{a}}$ & 37 & $2^{\mathrm{a}}$ & $3^{\mathrm{a}}$ & 5 & 0 & 79 & $27^{\mathrm{a}}$ \\
\hline 24 & 26 & $100^{\mathrm{a}}$ & $24^{\mathrm{a}}$ & $124^{\mathrm{a}}$ & $34^{\mathrm{a}}$ & 3 & 7 & 6 & 0 & $91^{\mathrm{a}}$ & $29^{\mathrm{a}}$ \\
\hline 27 & 25 & $23^{\mathrm{a}}$ & $8^{\mathrm{a}}$ & $31^{\mathrm{a}}$ & $25^{\mathrm{a}}$ & 4 & $3^{\mathrm{a}}$ & 5 & 0 & $152^{\mathrm{a}}$ & $19^{\mathrm{a}}$ \\
\hline 30 & $24^{\mathrm{a}}$ & $82^{\mathrm{a}}$ & $22^{\mathrm{a}}$ & $104^{\mathrm{a}}$ & $30^{\mathrm{a}}$ & 3 & $2^{\mathrm{a}}$ & $4^{\mathrm{a}}$ & $7^{\mathrm{a}}$ & $110^{\mathrm{a}}$ & $27^{\mathrm{a}}$ \\
\hline 32 & $13^{\mathrm{a}}$ & $20^{\mathrm{a}}$ & $2^{\mathrm{a}}$ & $22^{\mathrm{a}}$ & $33^{\mathrm{a}}$ & $2^{\mathrm{a}}$ & $1^{\mathrm{a}}$ & 5 & 2 & $113^{\mathrm{a}}$ & $30^{\mathrm{a}}$ \\
\hline 41 & 26 & $91^{\mathrm{a}}$ & 33 & $124^{\mathrm{a}}$ & 36 & 3 & 7 & 6 & 1 & 71 & 32 \\
\hline 43 & 25 & $84^{\mathrm{a}}$ & $23^{\mathrm{a}}$ & $107^{\mathrm{a}}$ & $32^{\mathrm{a}}$ & 3 & $1^{\mathrm{a}}$ & $4^{\mathrm{a}}$ & 1 & 71 & $24^{\mathrm{a}}$ \\
\hline
\end{tabular}

Nota. ALF (conhecimento do alfabeto); LEIT (fluência e acurácia de leitura de palavras e pseudopalavras); ESC (escrita de palavras e pseudopalavras); LE (LEIT + ESC); CFO (consciência fonológica); MTF (memória de trabalho fonológica); COP (cópia de formas); MV (memória visual); MVE (memória visuoespacial); RAN (nomeação automática rápida).

a Escores iguais ou mais de 1,65 DP abaixo da média do grupo controle específico de tarefa.

Tendo em vista a limitação de espaço, não mostraremos outros escolares que apresentaram baixo rendimento em algumas provas, mas que não fazem parte do GD, mas seus escores foram computados na sensitividade e especificidade (ver Tabela 4). A Tabela 3 mostra o grupo GD", isto é, os alunos do GD ${ }^{\mathrm{I}}$ que continuaram em defasagem acadêmica no ano seguinte e seus escores nas medições do TDCL ${ }^{\mathrm{II}}$.

Conforme mostra a Tabela 4, o conhecimento do alfabeto representou somente $53,8 \%$ dos escolares presentes no $\mathrm{GD}^{\mathrm{I}}$ (verdadeiros positivos), embora com excelente especificidade de $87,5 \%$ de verdadeiros negativos. Como esperado, as habilidades de leitura e escrita do TDCL ${ }^{\mathrm{I}}$ mostraram excelente sensitividade de 100 e $92,3 \%$ de verdadeiros positivos e especificidade de 93,7 e $96,8, \%$ de verdadeiros negativos, respectivamente, para o $\mathrm{GD}^{\mathrm{I}}$. O escore composto de leitura-escrita do TDCL alcançou 100\% de sensitividade e 96,8\% de especificidade. Para o grupo $\mathrm{GD}^{\mathrm{II}}$, as habilidades de leitura, escrita e leitura-escrita do TDCL ${ }^{\mathrm{I}}$ todas apresentaram sensitividade e especificidade de $100 \%$.

Com relação às provas não baseadas na linguagem escrita, a CFO do TDCL ${ }^{\mathrm{I}}$ apresentou a maior sensitividade de todas com 76,9 e $81 \%$ de verdadeiros positivos para o $\mathrm{GD}^{\mathrm{I}}$ e GD $\mathrm{GD}^{\mathrm{II}}$, respectivamente, e boa especificidade de 87,5 e $83,8 \%$, respectivamente. A memória de trabalho fonológica (MTF) e as provas não linguísticas do TDCL apresentaram, de um modo geral, uma sensitividade relativamente baixa, mas excelente especificidade. A MTF, a memória visual (MV), a memória visuoespacial (MVE) e a nomeação automática rápida (RAN) apresentaram muito boa especificidade variando de 97 a $94 \%$ para as duas primeiras, até $87 \%$ para as últimas, em relação a ambos os grupos $\mathrm{GD}^{\mathrm{I}}$ e $\mathrm{GD}^{\mathrm{II}}$, porém todas com sensitividades relativamente fracas, abaixo de $50 \%$. A COP apresentou uma sensitividade de 61,5 e $72,7 \%$ e especificidade de 84,3 e $83,8 \%$ para os grupos $\mathrm{GD}^{\mathrm{I}}$ e GD , respectivamente.

As FAE apresentaram exatamente a mesma sensitividade I e II da CFO que, como já mencionado, foi a segunda maior do $\mathrm{TDCL}^{\mathrm{I}}$, isto é, de 76,9 para o $\mathrm{GD}^{\mathrm{I}}$ e $81,8 \%$ para o $\mathrm{GD}^{\mathrm{II}}$, porém com especificidade mais baixa de 75 e $70,9 \%$ de verdadeiros negativos, respectivamente. Ambas as Tabelas 2 e 3 fornecem informações sobre o perfil cognitivo dos escolares de risco para TA com respeito às diferenças intraindividuais. A Tabela 2 revela que dos 13 escolares do GD', 10 apresentaram prejuízos na CFO no TDCL ${ }^{\mathrm{I}}$ (nove deles também prejudicados nas FAE), dos quais seis também tiveram déficit na RAN (escolares 5, 19, 24, 27, 30, 32), e quatro escolares apresentaram prejuízos na memória de trabalho visuoespacial (MTVE) (escolares 10, 18, 19, 30).

Dos três escolares do $\mathrm{GD}^{\mathrm{I}}$ que não apresentaram déficit na CFO do TDCL ${ }^{\mathrm{I}}$ (ver Tabela 2), um (escolar 24) saiu do grupo de risco no $3^{\circ}$ ano após as intervenções psicopedagógicas. O escolar 22 apresentou prejuízos no alfabeto e, mais importante, nas atividades FAE, notadamente uma medida da consciência fonológica (Andrade et al., 2011), e na MTF, apesar de repetente e um ano mais velho que seu grupo classe. Mesmo após intervenções pedagógicas e fonoaudiológicas, ele continuou com escore de CFO (37) muito próximo do 
Tabela 3. Escolares com Baixo Rendimento Escolar Identificados pela Avaliação Diagnóstica no Início do $3^{\circ} \mathrm{Ano}\left(\mathrm{GD}^{\mathrm{II}}\right)$ e seus Escores na Segunda Medição do Teste de Desempenho Cognitivo-Linguístico um Ano Após o Início do Estudo (TDCL ${ }^{\mathrm{II}}$ )

\begin{tabular}{ccccc}
\hline \multicolumn{5}{c}{ TDCL $^{\text {II }}$} \\
\hline GD $^{\text {II }}$ & Leitura $^{\text {II }}$ & CFO $^{\text {II }}$ & MTF $^{\text {II }}$ & RAN $^{\text {II }}$ \\
\hline 04 & $114^{\mathrm{b}}$ & $34^{\mathrm{a}}$ & 3 & 59 \\
\hline 05 & $96^{\mathrm{a}}$ & $29^{\mathrm{a}}$ & $2^{\mathrm{a}}$ & 76 \\
\hline 06 & $81^{\mathrm{a}}$ & 38 & 5 & $98^{\mathrm{a}}$ \\
\hline 10 & $101^{\mathrm{a}}$ & $21^{\mathrm{a}}$ & 4 & 76 \\
\hline 18 & $106^{\mathrm{a}}$ & $29^{\mathrm{a}}$ & 4 & 75 \\
\hline 22 & $97^{\mathrm{a}}$ & $35^{\mathrm{a}}$ & 4 & 71 \\
\hline 27 & $97^{\mathrm{a}}$ & 37 & 4 & 73 \\
\hline 30 & $79^{\mathrm{a}}$ & 37 & 5 & $108^{\mathrm{a}}$ \\
\hline 32 & $107^{\mathrm{a}}$ & $18^{\mathrm{a}}$ & $3^{\mathrm{a}}$ & 73 \\
\hline 43 & $93^{\mathrm{a}}$ & $32^{\mathrm{a}}$ & $2^{\mathrm{a}}$ & $99^{\mathrm{a}}$ \\
\hline $120^{\mathrm{b}}$ & $35^{\mathrm{a}}$ & 4 & $92^{\mathrm{a}}$ \\
\hline
\end{tabular}

Nota. LEIT (fluência e acurácia de leitura de palavras e pseudopalavras); CFO (consciência fonológica); MTF (memória de trabalho fonológica); RAN (nomeação automática rápida).

a Escores iguais ou mais de 1,65 DP abaixo da média do grupo controle. ${ }^{\text {b} E s c o r e s ~ a c i m a ~ d o ~ p o n t o ~ d e ~ c o r t e ~}(<106)$ no TDCL ${ }^{\mathrm{II}}$, mas muito abaixo do limite inferior do intervalo de confiança de $95 \% \quad(=129,79)$ do grupo controle específico de tarefa (GRE ${ }^{\mathrm{II}}$.

ponto de corte (36) no TDCL ${ }^{\text {II }}$. Esses dados sugerem fortemente que o escolar 22 possui déficit fonológicos capturados pelas FAE e MTF, mas não pela prova de CFO do TDCL. O terceiro (escolar 6) mostrou desempenho normal em CFO, mas apresentou severos prejuízos de MVE no TDCL ${ }^{\mathrm{I}}$ e de RAN no TDCL ${ }^{\mathrm{I}}$ e TDCL ${ }^{\mathrm{II}}$.

A Tabela 3 indica que os alunos do GD ${ }^{\text {II } q u e ~ m e s m o ~ a p o ́ s ~}$ um ano de intervenções psicopedagógicas ainda permanece- ram com baixo desempenho acadêmico na $\mathrm{AD}^{\mathrm{II}}$, formando os 11 alunos do GD ${ }^{\mathrm{II}}, 100 \%$ deles identificados pelos desempenhos em leitura-escrita (LE) do TDCL ${ }^{\mathrm{I}}$, e oito deles $(72,7 \%)$ pelas provas de leitura do TDCL ${ }^{\mathrm{II}}$ (LEIT $\left.{ }^{\mathrm{II}}\right)$. Embora dois alunos do $\mathrm{GD}^{\mathrm{II}}$ estivessem com escores de LEIT ${ }^{\mathrm{II}}$ acima do ponto de corte (escolares 4 e 43), eles ainda estavam abaixo do LIC de $95 \%$ do GRE e também prejudicados na CFO. Juntos, esses dados justificam a classificação desses escolares como BRE e, portanto, ainda de risco para TL na segunda avaliação diagnóstica $\left(\mathrm{AD}^{\mathrm{II}}\right)$.

Com relação às habilidades não baseadas na linguagem, a Tabela 3 revela que oito dos 11 escolares do $\mathrm{GD}^{\mathrm{II}}$ ainda continuavam com prejuízos de CFO. Entretanto, dois dos três escolares restantes do $\mathrm{GD}^{\mathrm{II}}$ que não apresentaram déficit na $\mathrm{CFO}^{\mathrm{II}}$ ainda continuaram com severos prejuízos de $\mathrm{RAN}^{\mathrm{II}}$ (ver Tabela 3).

\section{Discussão}

Consideramos importante fazer algumas observações em relação à amostragem do presente estudo. A amostra de 45 participantes corresponde aos termos de consentimento que retornaram de uma amostra maior de 69 alunos matriculados em quatro turmas do $2^{\circ}$ ano do ensino fundamental de uma escola particular e da qual 24,6\% (17 escolares) apresentavam queixas de baixo rendimento escolar (BRE) de acordo com o prontuário escolar e avaliação diagnóstica (Luckesi, 2005). Da amostra dos 45 participantes, 13 (28,8\%) fazem parte dos 17 escolares com BRE da amostra maior e compõem o grupo em defasagem no início do estudo $\left(\mathrm{GD}^{\mathrm{I}}\right)$. Ambos os percentuais de alunos com queixa são altamente consistentes com trabalhos recentes indicando que $30 \%$ da população do $1^{\circ}$ ao $5^{\circ}$ anos, incluindo rede pública e privada, apresentam queixas (Alves \& Ribeiro, 2011; Bicalho \& Alves, 2010; Refundini, Martins \& Capellini, 2010). Não obstante uma das limitações do presente estudo ser o tamanho reduzido da amostra, a consistência entre a prevalência BRE observada e a literatura sugere que se trata de uma amostra válida para um estudo piloto.

Utilizando como referência o grupo de 13 alunos em defasagem acadêmica (ver Tabela 2), investigou-se a sensiti-

Tabela 4. Acurácia das Provas do TDCL ${ }^{\mathrm{I}}$ e das FAE na Identificação dos Escolares em Defasagem Acadêmica no Início do $2^{\circ}$ Ano (Sensitividade I e Especificidade I) e do $3^{\circ}$ Ano (Sensitividade II e Especificidade I)

Provas do Teste de Desempenho Cognitivo-Linguístico I (TDCL I)

\begin{tabular}{|c|c|c|c|c|c|c|c|c|c|c|c|}
\hline & ALF & LEIT & ESC & LE & CFO & MTF & COP & MV & MVE & RAN & FAE \\
\hline Sensitividade I & 53,8 & 100 & 92,3 & 100 & 76,9 & 30,7 & 61,5 & 30,7 & 38,4 & 46,1 & 76,9 \\
\hline Especificidade I & 87,5 & 93,7 & 96,8 & 96,8 & 87,5 & 96,8 & 84,3 & 93,7 & 87,5 & 87,5 & 75 \\
\hline Sensitividade II & 63,6 & 100 & 100 & 100 & 81,8 & 36,3 & 72,7 & 36,3 & 45,4 & 54,5 & 81,8 \\
\hline Especificidade II & 91,1 & 88,2 & 94,1 & 91,1 & 85,2 & 97,0 & 85,2 & 94,1 & 88,2 & 88,2 & 73,5 \\
\hline
\end{tabular}

Nota. Sensitividade (verdadeiros positivos (percentual dos escolares de risco detectados); Especificidade (verdadeiros negativos (percentual dos escolares fora de risco não detectados); TDCL ${ }^{\mathrm{I}}$ (Teste de Desempenho Cognitivo-Linguístico, aplicado no início do estudo); FAE (Ferramentas Alternativas do Educador, aplicadas somente no início do estudo); ALF (conhecimento do alfabeto); LEIT (fluência e acurácia de leitura de palavras e pseudopalavras); ESC (escrita de palavras e pseudopalavras); LE (LEIT + ESC); CFO (consciência fonológica); MTF (memória de trabalho fonológica); COP (cópia de formas); MV (memória visual); MVE (memória visuoespacial); RAN (nomeação automática rápida). 
vidade e especificidade das provas do Teste de Desempenho Cognitivo-Linguístico (TDCL) (Capellini \& Smythe, 2008) e de tarefas pedagógicas coletivas denominadas de Ferramentas Alternativas do Educador (FAE) na identificação de escolares de risco para transtornos de leitura (TL), com foco nas provas de caráter coletivo e em um contexto educacional real com uma abordagem de RI (resposta à intervenção). As provas individuais do TDCL serviram como referência de teste padronizado para as habilidades de linguagem escrita e investigação dos principais componentes cognitivos subjacentes à sua aquisição.

As provas de leitura e escrita do $\mathrm{TDCL}^{\mathrm{I}}$ (início do $2^{\circ}$ ano) apresentaram excelente acurácia na identificação dos escolares de risco de ambos os grupos GD ${ }^{\mathrm{I}}$ e GD $\mathrm{GI}^{\mathrm{II}}$ (ver Tabela 4), com sensitividade ao $\mathrm{GD}^{\mathrm{I}}$ de $92,3 \%$ para a escrita (ESC) e de $100 \%$ para a leitura (LEIT) e para a leitura-escrita (LE), todas alcançando $100 \%$ para o $\mathrm{GD}^{\mathrm{II}}$, e todas com excelente especificidade para ambos o $\mathrm{GD}^{\mathrm{I}}$ e $\mathrm{GD}^{\mathrm{II}}$ (média de $6 \%$ de falsos positivos). Esses resultados sugerem a eficiência da avaliação diagnóstica e a importância da equipe pedagógica da escola no trabalho de identificação de escolares de risco (Capellini et al., 2004).

As FAE, além de se correlacionarem com a linguagem escrita e com a CFO do TDCL ${ }^{\mathrm{I}}$ em estudo anterior (Andrade et al., 2011), apresentaram exatamente a mesma sensitividade de $76,9 \%$ da CFO para o GD ${ }^{\mathrm{I}}$, a qual aumentou para $81,8 \%$ no caso do GD ${ }^{\text {II }}$. Esse resultado revela o potencial das FAE como ferramenta coletiva de rastreamento de escolares de risco, capaz de substituir as provas demoradas e antieconômicas dos testes individuais de CFO e abre caminho para investigações futuras confirmatórias. Argumenta-se que os erros de falsos negativos (baixa especificidade) são muito mais sérios do que os falsos positivos porque privam escolares de risco de receberem a intervenção (Barth et al., 2008), e que a grande maioria dos estudos tem encontrado estimativas de falsos negativos variando de $10 \%$ a $60 \%$ (Vellutino et al., 2008). Em nosso estudo, as provas do TDCL e as FAE variaram de 0 a $25 \%$ de falsos negativos, e as provas coletivas de escrita do TDCL juntamente com as FAE são exemplos motivadores na busca por ferramentas coletivas de identificação do risco para TL.

A despeito de sua baixa sensitividade, os testes individuais de memória de trabalho e RAN do TDCL apresentaram excelente especificidade. A memória de trabalho visuoespacial (MVE) foi a única a capturar os déficits atencionais de cinco escolares do GD ${ }^{\mathrm{I}}$ e GD ${ }^{\mathrm{II}}$ (ver Tabelas 2 e 3 ) que apresentavam comportamento típico de TDAH, de acordo com o prontuário escolar e com a Escala de Transtorno de Déficit de Atenção/Hiperatividade - versão para professores (Benczik, 2000). Esse dado é consistente com a literatura recente sobre o TDAH (Castellanos et al., 2006), segundo a qual prejuízos na memória visuoespacial são a principal evidência dos déficits atencionais (Castellanos et al., 2006; Martinussen \& Tannock, 2006). Um desses escolares não apresentava déficits fonológicos, mas somente prejuízo na memória de trabalho fonológica e sérios prejuízos na RAN (o segundo mais prejudicado de todo o GD). Esses resultados são altamente consistentes com evidências recentes de que ambos, a inatenção e o prejuízo na consciência fonológica, são os principais fatores etiológicos de origem genética subjacentes aos déficits de leitura, mas os quais constituem mecanismos independentes que frequentemente ocorrem associados na comorbidade TDAH-dislexia (Willcutt et al., 2010).

De acordo com a $\mathrm{AD}^{\mathrm{II}}$, o número de escolares de risco caiu para 11 alunos (ver Tabela 3). Embora no segundo ano do experimento a amostra tivesse quatro alunos a menos $(n=41)$ nenhum desses ausentes fazia parte do grupo de risco, portanto podemos considerar que um ano depois, o percentual de escolares de risco caiu de $28,8 \%$ para $24,4 \%$. A Tabela 3 mostra os escolares que, de fato, são de risco para TL, pois continuaram com problemas de aprendizagem após um ano de intervenções, bem como permite avaliar quais os principais prejuízos cognitivos subjacentes às suas dificuldades persistentes de leitura os quais, consistentemente com a literatura (Fletcher et al., 2011; Vellutino et al., 2004, 2008), foram de consciência fonológica. Entretanto, dois escolares (6 e 27) apresentaram severos prejuízos de leitura preservando a CFO, explicados somente pelos seus prejuízos de RAN. O escolar 6 mostrou claros prejuízos atencionais e de RAN, com preservação da CFO, bem como padrão comportamental típico do TDA/H de acordo com a escala de Benczik (2000), quadro consistente com evidências crescentes de que a desatenção está associada a déficits de nomeação rápida e memória de trabalho e representa um fator causal importante de TL independente da consciência fonológica (Shaywitz \& Shaywitz, 2008; Willcutt et al., 2010). O escolar 27 apresentou escores normais de CFO no TDCL ${ }^{\mathrm{II}}$ e nunca apresentou sinais de desatenção e/ou hiperatividade, nem nos prontuários e nem em nenhuma das medições, constituindo-se num caso específico que corrobora a hipótese do duplo-déficit, segundo a qual, os déficits de consciência fonológica e de nomeação rápida (velocidade de processamento) são independentes e subjacentes ao TL (Wolf \& Bowers, 1999).

\section{Conclusão}

Este estudo, até onde sabemos, é a primeira investigação no Brasil de um modelo de resposta à intervenção. Não obstante seu tamanho reduzido, as características da amostra são consistentes com amostras muito maiores e as tarefas coletivas testadas apresentaram boa sensitividade e especificidade, indicando o grande potencial de investigações voltadas para o desenvolvimento de ferramentas de baixo custo e uso prático em contexto escolar na identificação precoce, particularmente dos transtornos da atenção e de aprendizagem da leitura. Também, as ferramentas e os procedimentos testados podem ser considerados como um modelo de RI ampliado, pois incluem, além da avaliação da linguagem escrita, avaliações das habilidades de processamento fonológico e visuoespaciais que permitem a caracterização do perfil cognitivo-linguístico individual do escolar de risco, um aspecto essencial para a adequação das intervenções às necessidades individuais do aluno, e o qual não é contemplado no modelo tradicional. Esses resultados descortinam um promissor horizonte de pesquisa para a área. 


\section{Referências}

Almeida, P. M., \& Wickerhauser, H. (1991). O critério ABA/ ABIPEME: Em busca de uma atualização. São Paulo: LPM/ Burke e Marplan.

Alves, L. M., Mousinho, R., \& Capellini, S. (2011). Dislexia. Novos temas, novas perspectivas. Rio de Janeiro: Wak Editora.

Alves, L. M., \& Ribeiro, M. M. (2011). Desempenho em memória de trabalho em escolares com e sem queixas de alterações de aprendizagem. Tecer, 4, 54-65.

Alves L. M., Siqueira, C. M., Lodi, D. F., \& Araújo, M. C. M. F. (2011). Introdução à dislexia do desenvolvimento. In L. M. Alves, R. Mousinho \& S. A. Capellini (Eds.), Dislexia. Novos temas, novas perspectivas (pp. 21-40). Rio de Janeiro: Wak Editora.

American Psychiatric Association. (2002). DSM-IV-TR: Manual diagnóstico e estatístico de transtornos mentais (4a ed.). Porto Alegre: Artmed.

Andrade, O. V. C. A., Prado, P. S. T., \& Capellini, S. A. (2011). Desenvolvimento de ferramentas pedagógicas para identificação de escolares de risco para a dislexia. Revista Psicopedagogia, 28(85), 14-28.

Barth, A. E., Stuebing K. K., Anthony, J. L., Denton, C. A., Mathes, P. G., Fletcher, J. M., \& Francis, D. J. (2008). Agreement among response to intervention criteria for identifying responder status. Learning and Individual Differences, 18, 296-307.

Benczik, E. B. P. (2000). Manual da escala de transtorno de déficit de atenção/ hiperatividade: versão para professores. São Paulo: Casa do Psicólogo, 2000.

Bicalho, L. G. R., \& Alves, L. M. (2010). A nomeação seriada rápida em escolares com e sem queixas de problemas de aprendizagem em escola pública e particular. Revista do Centro de Especialização em Fonoaudiologia Clínica, 12, 608-616.

Capellini, S. A., \& Conrado, T. L. B. C. (2009). Desempenho de escolares com e sem dificuldades de aprendizagem de ensino particular em habilidade fonológica, nomeação rápida, leitura e escrita. Revista do Centro de Especialização em Fonoaudiologia Clínica, 11, 183-193.

Capellini, S. A., Sampaio, M. N., Matsuzawa, M. T., Oliveira, A. M., Fadini, C. C., \& Martins, M. A. (2009). Protocolo de identificação precoce dos problemas de leitura: Estudo preliminar com escolares de $1^{\circ}$ ano escolar. Revista Psicopedagogia, 26(80), 367-375.

Capellini, S. A., \& Smythe, I. (2008). Protocolo de avaliação de habilidades cognitivo-linguísticas: Livro do profissional e do professor. Marília: Fundepe.

Capellini, S. A., Tonelotto, J. M. F., \& Ciasca, S. M. (2004). Medidas de desempenho escolar: avaliação formal e opinião de professores. Estudos em Psicologia, 21, 79-90.

Castellanos, F. X., Sonuga-Barke, E. J., Milham, M. P., \& Tannock, R. (2006). Characterizing cognition in ADHD: Beyond executive dysfunction. Trends in Cogntive Sciences, 10(3), 117-123.

Catts, H. W., Fey, M. E., Zhang, X., \& Tomblin, J. B. (2001). Estimating the risk of future reading difficulties in kindergarten children: A research-based model and its clinical implementation. Language, Speech, and Hearing Services in Schools, 32, 38-50.

Ciasca, S. M. (2004). Distúrbios de aprendizagem: proposta de avaliação interdisciplinar. São Paulo: Casa do Psicólogo.
Demo P. (2007). Escola pública e escola particular: Semelhanças de dois imbróglios educacionais. Ensaio: Avaliação e Politicas Públicas em Educação, 15(55), 181-206.

Dombrowski, S. C., Kamphaus, R. W., \& Reynolds, C. R. (2004). After the demise of the discrepancy: Proposed approach to learning disabilities classification. Professional Psychology: Research and Practice, 35(4), 364-372.

Fletcher, J. M., Stuebing, K. K., Barth, A. E., Denton, C. A., Cirino, P. T, Francis, D. J., \& Vaughn, S. (2011). Cognitive correlates of inadequate response to reading intervention. School Psychology Review, 40, 3-22.

Fletcher, J., \& Vaughn, S. (2009). Response to intervention: Preventing and remediating academic difficulties. Child Development Perspectives, 3, 30-37.

Fuchs, D., \& Fuchs, L. S. (2006). Introduction to response to intervention: What, why, and how valid is it? Reading Research Quarterly, 41, 93-99.

Hinshaw, S. P. (1992). Academic underachievement, attention deficits, and aggression comorbidity and implications for intervention. Journal of Consulting and Clinical Psychology, 60, 893-903.

Luckesi, C. C. (2005). Avaliação da aprendizagem na escola: Reelaborando conceitos e criando a prática ( $2^{\mathrm{a}} \mathrm{ed}$.). Salvador: Malabares Comunicações e Eventos.

Martinussen, R., \& Tannock, R. (2006). Working memory impairments in children with attention-deficit hyperactivity disorder with and without comorbid language learning disorders. Journal of Clinical and Experimental Neuropsychology, 28, 1073-94.

Navas, A. L. (2011). Por que prevenir é melhor que remediar quando se trata de dificuldades de aprendizagem. In L. M. Alves, R. Mousinho \& S. A. Capellini (Eds.), Dislexia: novos temas, novas perspectivas (pp. 41-53). Rio de Janeiro: Wak Editora.

Ramus, F., Rosen, S., Dakin, S. C., Day, B. L., Castellote, J. M., White, S., \& Frith, U. (2003). Theories of developmental dyslexia: Insights from a multiple case study of dyslexic adults. Brain, 126, 841-65.

Refundini, D. C., Martins, M. A., \& Capellini S. A. (2010). Treinamento da correspondência grafema-fonema em escolares de risco para a dislexia. Revista Psicopedagogia, 27 (83), 191-201.

Reynolds, C. R., \& Shaywitz, S. E. (2009). Response to Intervention: Ready or not? Or, from wait-to-fail to watch-them-fail. School Pschology Quaterly, 24, 130-145.

Shanahan, M. A., Pennington, B. F., Yerys, B. E., Scott, A., Boada, R., Willcutt, E. G., . . . DeFries, J. C. (2006). Processing speed deficits in attention deficit/hyperactivity disorder and reading disability. Journal of Abnormal Child Psychology, 34, 585-602.

Shaywitz, S. E., Morris, R., \& Shaywitz, B. A. (2008). The education of dyslexic children from childhood to young adulthood. Annual Review of Psychology, 59, 451-475.

Shaywitz, S. E., \& Shaywitz, B. A. (2008). Paying attention to reading: The neurobiology of reading and dyslexia. Development and Psychopathology, 20, 1329-1349.

Silva, P. A., \& Santos, F. H. (2011). Discalculia do desenvolvimento: Avaliação da representação numérica pela ZAREKI-R. Psicologia: Teoria e Pesquisa, 27(2), 169-177. 
Siqueira, C. M., Gurgel-Giannetti, J., Araújo, M. C. F. M., Lodi, D. F., Alves, J. F. M., Avelar, K., \& Alves, L. M. (2011). Transtorno de déficit de atenção/hiperatividade e transtornos de aprendizagem. In L. M. Alves, R. Mousinho, \& S. A. Capellini (Eds.), Dislexia: Novos temas, novas perspectivas (pp. 283301). Rio de Janeiro: Wak Editora.

Vellutino, F. R., Fletcher, J. M., Snowling, M. J., \& Scanlon, D. M. (2004). Specific reading disability (dyslexia): What have we learned in the past four decades? Journal of Child Psychiatry and Psychology and Allied Disciplines, 45, 2-40.

Vellutino, F. R., Scanlon, D. M., Zhang, H., \& Schatschneider, C. (2008). Using response to kindergarten and first grade intervention to identify children at-risk for long-term reading difficulties. Reading and Writing: An Interdisciplinary Journal, 21, 437-480.
Willcutt, E. G., Betjemann, R. S., McGrath, L. M., Chhabildas, N. A., Olson, R. K., DeFries, J. C., \& Pennington, B. (2010). Etiology and neuropsychology of comorbidity between RD and ADHD: the case for multiple-deficit models. Cortex, 46, 1345-1361.

Wolf, M., \& Bowers, P. G. (1999). The double-deficit hypothesis for the developmental dyslexias. Journal of Educational Psychology, 91, 415-438.

Recebido em 04.03.2012 Primeira decisão editorial em 04.06.2012

Versão final em 22.08.2012

Aceito em 31.08.2012

\section{$43^{a}$ REUNIÃO ANUAL DA SOCIEDADE BRASILEIRA DE PSICOLOGIA}

Período: 23 a 26 outubro de 2013

Local: Aracaju, SE

http://www.ra.sbponline.org.br/ 